\title{
On the second-order characteristics of marked point processes
}

\author{
MARTIN SCHLATHER \\ Department of Mathematics and Statistics, Lancaster University, Lancaster LA1 4YF, United \\ Kingdom.E-mail: Martin.Schlather@uni-bayreuth.de
}

\begin{abstract}
New definitions for the second-order characteristics of marked point processes are presented. Nonreduced moment measures are involved in obtaining consistency with analogous definitions in the random field context. A certain $\sigma$-algebra replaces the current assumptions on stationarity and isotropy so that the characteristics are still well defined even if such assumptions do not hold. The new definitions and the present ones coincide for positive arguments if the marked point process is simple, stationary and isotropic, and if the second-order product density exists. The different second-order characteristics given in the literature are discussed. A renaming of one of the characteristics is suggested since distinct characteristics have hitherto been known under identical names. Furthermore, it is shown that arbitrary measurable functions with compact support can appear as second-order characteristics of marked point processes.
\end{abstract}

Keywords: mark correlation function; mark covariance function; mark variogram; marked point process; moment measure; second-order characteristic

\section{Introduction}

Stationary and isotropic marked point processes form useful models in geology (Wen and Sinding-Larsen 1997), medicine (Diggle 1983) and forestry (Gavrikov and Stoyan 1995; Goulard et al. 1995; Penttinen et al. 1992; and Stoyan and Stoyan 1994). Stoyan et al. (1995) give an introduction to general marked point processes. Important data-analytical tools for investigating such processes are the second-order characteristics for both the locations and the marks. This paper deals with theoretical aspects of second-order characteristics for marks.

Unnormed second-order characteristics are the mark variogram of Cressie (1993) and the mark covariance functions of Cressie (1993) and Stoyan (1984a). Normed second-order characteristics are Isham's (1985) mark correlation function and Stoyan's (1984b) $\mathrm{kmm}_{\mathrm{m}}$ function; see also Penttinen and Stoyan (1989). Each characteristic is a function of the distance between two points of the point process, i.e. the domain is the positive real axis. Its behaviour is of interest on the whole domain in order to characterize the marked point process; see Wälder and Stoyan (1996; 1998).

In Section 2, the present definitions for the characteristics are recalled and their names are discussed. Two theorems highlight that the behaviour of a second-order characteristic of a marked point process differs from that of a random field. Theorem 2.1 shows that, under some minor constraints, any measurable function with compact support can appear as a 
second-order characteristic for a marked point process. Theorem 2.2 shows that there are two marked point processes with the same $k_{m m}$-function but with quite different mark correlation functions, if the $k_{m m}$-function is non-negative on a finite interval and identically 1 elsewhere.

The present definitions may lead to functions that are not well defined at the origin or where the value at the origin is inconsistent with the definitions given in the random field context. Therefore, new definitions are suggested in Section 3. They are introduced in two ways. The first approach, intuitive and geometrically based, still needs stationarity assumptions but does not require isotropy. It leads to characteristics that are fully consistent with those given in the random field context. In the second approach the definitions are based on a certain $\sigma$-algebra. Stationarity assumptions are then not required in order to obtain characteristics that depend on the distance between two points only.

In Section 4, two examples of marked point processes and their second-order characteristics are given. Secion 5 provides the proofs for and some extensions of the statements in Section 2.

\section{Present definitions and properties}

Different notations have been used in the literature for the various second-order characteristics. Here, we follow the notation of Stoyan and Stoyan (1994).

\subsection{The present definitions}

In contrast to conventional geostatistics, a second-order characteristic of a marked point process should be interpreted as a conditional quantity. For example, the value of Isham's mark correlation function at a point $r$ equals the (usual) correlation coefficient between two marks given that the corresponding points are a distance $r$ apart.

Consider a bounded sampling window. Then, in general, the probability of finding a pair of points at a fixed distance $r$ is zero. Therefore it is difficult to define conditional quantities directly. Instead, a measure $\alpha_{f}^{(2)}$ is considered, in the same way that certain integrals are considered in the definition of an ordinary conditional expectation. The derivative $\rho_{f}^{(2)}$ of $\alpha_{f}^{(2)}$ (with respect to Lebesgue measure) depends on the intensity of the marked point process. As only the marks are of interest, a normalized version $\kappa_{f}$ of $\rho_{f}^{(2)}$ is used to define the second-order characteristics.

Let $\Phi$ be a stationary and isotropic, simple marked point process on $\mathbb{R}^{d}$ with nonnegative marks, and denote the Borel $\sigma$-algebra of $\mathbb{R}^{d}$ by $\mathscr{B}^{d}$. For an arbitrary measurable non-negative function $f$ on $\mathbb{R}^{2}$, the measure $\alpha_{f}^{(2)}$ on $\mathbb{R}^{2 d}$ is defined by

$$
\alpha_{f}^{(2)}\left(B_{1} \times B_{2}\right)=\mathrm{E} \sum_{\left[x_{1} ; m_{1}\right],\left[x_{2} ; m_{2}\right] \in \Phi}^{\neq} f\left(m_{1}, m_{2}\right) \mathbf{1}_{B_{1}}\left(x_{1}\right) \mathbf{1}_{B_{2}}\left(x_{2}\right), \quad B_{1}, B_{2} \in \mathscr{B}^{d}
$$

see Penttinen and Stoyan (1989). (The symbol $\sum^{\neq}$signifies that the sum runs only over pairs of marked points $\left[x_{1} ; m_{1}\right]$ and $\left[x_{2} ; m_{2}\right]$ with $x_{1} \neq x_{2}$.) If $\alpha_{f}^{(2)}$ has a Lebesgue density 
$\rho_{f}^{(2)}\left(y_{1}, y_{2}\right)$ then $\rho_{f}^{(2)}$ depends only on the distance $r$ between the points $y_{1}$ and $y_{2}$ because of the invariance assumptions. If $f$ is identically 1 , then the index $f$ is dropped, i.e. we write $\alpha^{(2)}$ and $\rho^{(2)}$ for short. The measure $\alpha^{(2)}$ is then the reduced second moment measure for the corresponding unmarked point process $\Phi^{\prime}$ of $\Phi$, and $\rho^{(2)}$ is the second-order product density of $\Phi^{\prime}$. Let $\bar{m}$ be the mean mark of $\Phi$, and define

$$
\kappa_{f}(r)=\frac{\rho_{f}^{(2)}(r)}{\rho^{(2)}(r)},
$$

where $f$ stands for one of the following functions:

$$
\begin{aligned}
& e\left(m_{1}, m_{2}\right)=m_{1}, \\
& c\left(m_{1}, m_{2}\right)=m_{1} m_{2}
\end{aligned}
$$

or

$$
v\left(m_{1}, m_{2}\right)=m_{1}^{2} \text {. }
$$

Instead of the functions $e$ and $v$, the functions $e^{*}\left(m_{1}, m_{2}\right)=m_{2}$ and $v^{*}\left(m_{1}, m_{2}\right)=m_{2}^{2}$ respectively may be used as they lead to the same $\kappa$-functions, i.e. $\kappa_{e} \equiv \kappa_{e^{*}}$ and $\kappa_{v} \equiv \kappa_{v^{*}}$. Then, the mark variogram $\gamma$ (Cressie 1993), the mark covariance function cov (Cressie 1993), Stoyan's mark covariance function $\operatorname{cov}_{S}$ (Stoyan 1984a), Stoyan's $k_{m m}$-function (Stoyan 1984b), and Isham's mark correlation function cor (Isham 1985) are defined, for positive $r$, as

$$
\begin{aligned}
\gamma(r) & =\kappa_{v}(r)-\kappa_{c}(r), \\
\operatorname{cov}(r) & =\kappa_{c}(r)-\left(\kappa_{e}(r)\right)^{2}, \\
\operatorname{cov}_{S}(r) & =\kappa_{c}(r)-\bar{m}^{2}, \\
k_{m m}(r) & =\kappa_{c}(r) / \bar{m}^{2}, \\
\operatorname{cor}(r) & =\frac{\kappa_{c}(r)-\left(\kappa_{e}(r)\right)^{2}}{\kappa_{v}(r)-\left(\kappa_{e}(r)\right)^{2}},
\end{aligned}
$$

respectively. The value at the origin of each function is defined as the limit value for $r \downarrow 0$, if the function is defined for all positive values of $r$; see Stoyan et al. (1995, pp. 113ff.), Stoyan and Stoyan (1994, pp. 291ff.), Stoyan (1990) and Isham (1985).

\subsection{Properties and comments}

A second-order characteristic for a stationary marked point process is only given Lebesgue almost surely, since Lebesgue densities are involved in the definition of $\kappa_{f}$. Furthermore, the function $\kappa_{f}(r)$, and therefore all the characteristics, are undefined for distances $r$ that do not appear as inter-point distances of the marked point process. For example, if the point process 
is a hard-core process with hard-core radius $R$, then $\kappa_{f}$ is undefined on the interval $[0, R]$. See Stoyan et al. (1995) for an introduction to hard-core processes.

Distinct characteristics have hitherto been known under identical names. In this paper, one of the characteristics has been renamed, namely Stoyan's $k_{m m}$-function, having been introduced into the literature as the mark correlation function. Isham (1985) introduced another mark correlation function, cor, whose definition is closer to the definition of the correlation function for a random field. Therefore, Isham's definition rather than Stoyan's has been adopted here. Two different definitions for a mark covariance function were introduced by Stoyan (1984a) and Cressie (1993); cf. (8.7.18) and (8.7.19) in Cressie (1993). Cressie's covariance function cov is compatible with the classical definition of covariance. That is to say, the value of the function cov at a point $r$ can be interpreted as the conditional covariance of the marks at two points a distance $r$ apart. Stoyan's $\operatorname{cov}_{S}$ will not be considered in the following since, given $\bar{m}, \operatorname{cov}_{S}$ is obtained by a linear transformation of the $k_{m m}$-function.

Stoyan's $k_{m m}$-function equals the conditional, non-centred moment measure $\kappa_{c}$ up to the normalizing factor $\bar{m}^{2}$. It carries different information than the mark correlation function or the mark covariance function since the function $\kappa_{e}$ that appears in the definition of cor and cov is, in general, not identically constant for a marked point process (in contrast to the corresponding quantity for a random field); see Example 4.1 below. Stoyan's $k_{m m}$-function has been introduced for marked point processes with non-negative marks. Its definition can be extended to marked point processes with arbitrary real valued marks if $\bar{m} \neq 0$; see Section 3.4. Henceforth, it will always be tacitly assumed that $\bar{m} \neq 0$ whenever Stoyan's $k_{m m}$-function is used to characterize a marked point process with arbitrary marks.

The following two theorems, whose proofs are postponed to Section 5, give some insight into the behaviour of the second-order characteristics of marked point processes. They hold for Euclidean spaces of arbitrary positive dimension. Theorem 2.1 shows that, from a practical point of view, almost any function (with compact support) can appear as a secondorder characteristic of a marked point process. This behaviour is in sharp contrast to the confining property of positive definiteness for a second-order characteristic of a stationary random field. Theorem 2.2 shows in particular that Stoyan's $k_{m m}$-function and the mark correlation function are not linearly dependent with a non-negative factor of proportionality, unlike the corresponding characteristics for a stationary random field.

Theorem 2.1. Let $f$ be an arbitrary real-valued measurable function on $(0, \infty)$ with compact support $K$. Then, there exist stationary and isotropic marked point processes with nonnegative marks such that, for positive arguments,

(a) $f=\operatorname{cov}$;

(b) $f=k_{m m}-1$ if $f \geqslant-1$;

(c) $f=$ cor if $f$ takes values only in $[-1,1]$.

If $f \geqslant 0$ then there exists a stationary marked point process with non-negative marks such that $f=\gamma$ for positive arguments lying in $K$.

Theorem 2.2. Let $R$ be a finite positive number and let $f(r)$ be a measurable function on 
$(0, \infty)$ that is identically 1 for $r>R$. Then, the function $f$ is positive if and only if there exist two stationary and isotropic marked point processes $\Phi_{1}$ and $\Phi_{2}$ with non-negative marks, corresponding $k_{m m}$-functions $k_{m m}^{(1)}$ and $k_{m m}^{(2)}$, and corresponding mark correlation functions $\operatorname{cor}^{(1)}$ and cor $^{(2)}$ such that

$$
\begin{aligned}
k_{m m}^{(1)}(r) & =k_{m m}^{(2)}(r)=f(r), \quad r>0, \\
\operatorname{cor}^{(1)}(r) & =-\operatorname{cor}^{(2)}(r), \quad r>0,
\end{aligned}
$$

and $\operatorname{cor}^{(1)}(r) \neq 0$ if $0<r \leqslant R$.

\section{New definitions}

New definitions, motivated in Section 3.1, are given in the traditional notation of stochastic geometry in Section 3.2. Estimators are introduced in Section 3.3. In Section 3.4, the definitions are generalized and given in the terminology of probability theory. Some properties of the second-order characteristics are briefly discussed in Section 3.5.

\subsection{Motivation}

An important tool for characterizing an unmarked point process is Ripley's $K$-function; see Stoyan and Stoyan (1994) and Stoyan et al. (1995). This function was originally defined only for stationary and isotropic point processes; see Ripley (1977) or Ripley (1981, Section 8.1). The isotropy assumption is often dropped, (see, for example, Cressie 1993, Sections 8.2.6 and 8.3.5), and even stationarity cannot be always assumed (Diggle et al. 1991), but the $K$ function is used as if the stationarity and isotropy assumptions held. Cressie (1993, p. 618) states that 'versions [of the $K$-function] for more general [non-stationary] processes are clearly needed'. Like the $K$-function, the second-order characteristics given in Section 2.1 are strictly defined only for stationary and isotropic marked point processes. New definitions, providing a theoretical framework for investigating (not necessarily stationary or isotropic) marked point processes, will indirectly give a justification for the use of characteristics like Ripley's $K$-function when stationarity or isotropy assumptions do not hold. Another way of extending Ripley's $K$-function to inhomogeneous point processes is given by Baddeley et al. (1998).

The extension of the present definitions to anisotropic marked point processes is not straightforward if we wish to keep the condition that the second-order characteristics depend on the distance only. The reason is that the present definitions have been made from a model-based point of view involving the second-order product density $\rho^{(2)}$ as the key element. The latter depend only on the distance between two points (and thus the characteristics can be defined) if and only if the marked point process is stationary and isotropic. Changing the point of view towards statistical analysis provides the solution. From the standpoint of statistics, the characteristics under consideration are 'summaries' of the marked point process. Such a summary can be based on and exactly defined by a 
corresponding $\sigma$-algebra. The summary, and thus the $\sigma$-algebra, must be chosen according to the aim of the investigation. When data sets of different marked point processes are to be compared, it might be sufficient to use a statistic that depends only on the distance, even if the marked point processes might not be isotropic. (Diggle et al. (1991) provide an example in the case of unmarked point processes.) However, such a statistic would not be suitable for investigating directional features.

In this paper, the choice of the $\sigma$-algebra will not be discussed. It will always be assumed throughout that a suitable $\sigma$-algebra is the very simple one leading to characteristics that depend on the distance.

The definition of $\kappa_{f}$ in (2.2) involves the Lebesgue density $\rho^{(2)}$ of the measure $\alpha^{(2)}$. An example of a point process where $\alpha^{(2)}$ does not have a Lebesgue density is a Poisson cluster process where the daughter process consists of two points at a fixed distance $r_{0}$; see Stoyan et al. (1995, pp. 150ff.) for an introduction to Poisson cluster processes. A closer look at the definition of $\kappa_{f}$ in (2.2) shows that such kinds of processes can be dealt with. Let $\lambda$ be the $2 d$-dimensional Lebesgue measure; then

$$
\begin{aligned}
\kappa_{f}(r) & =\frac{\rho_{f}^{(2)}(r)}{\rho^{(2)}(r)}=\frac{\rho_{f}^{(2)}\left(x_{1}, x_{2}\right)}{\rho^{(2)}\left(x_{1}, x_{2}\right)}=\frac{\mathrm{d} \alpha_{f}^{(2)}\left(x_{1}, x_{2}\right) / \mathrm{d} \lambda\left(x_{1}, x_{2}\right)}{\mathrm{d} \alpha^{(2)}\left(x_{1}, x_{2}\right) / \mathrm{d} \lambda\left(x_{1}, x_{2}\right)} \\
& =\frac{\mathrm{d} \alpha_{f}^{(2)}\left(x_{1}, x_{2}\right)}{\mathrm{d} \alpha^{(2)}\left(x_{1}, x_{2}\right)},
\end{aligned}
$$

where $r=\left\|x_{1}-x_{2}\right\|$. The right-hand side is well defined even if the second-order product density does not exist.

The definition of the characteristics at the origin is not satisfactory for two reasons. Firstly, it is tacitly assumed that the limit of a function given by (2.3), (2.4), (2.6) or (2.7) exists as $r \downarrow 0$, if the function is defined for all positive values of $r$. Theorem 2.1, however, shows that this assumption does not hold in general and it does not help to redefine the function on a null set. Such a redefinition is sufficient for a measurable second-order characteristic of a random field to ensure the existence of that limit; see Sasvári (1994, Theorem 3.1.2).

Secondly, the definitions cause inconsistencies at the interface between random field theory and point process theory. The random field model of Mase (1996) and Wälder and Stoyan $(1996 ; 1997)$ is at this interface and is characterized by marks that are independent of the locations. It is a basic model for marked point processes in the way that the Poisson process is for unmarked point processes. Wälder and Stoyan's $(1996$; 1997) constructive definition of the random field model is the following. Let $\Xi$ be a stationary and isotropic random field and $\Phi$ a simple, unmarked, point process that is independent of $\Xi$. Then, the random field model $\Psi$ is given by

$$
\Psi=\bigcup_{x \in \Phi}[x ; \Xi(x)]
$$

In a geostatistical context, the points of $\Psi$ describe deterministic or stochastic sampling locations and the marks of $\Psi$ are the measured values of the random field $\Xi$. Thus, the 
random field model can also be characterized from a geostatistical point of view. However, a second-order characteristic for the random field coincides with that of the marked point process only for positive arguments, but not necessarily at the origin. For example, in the case of the nugget-effect model (see Wackernagel 1995, p. 218), the mark covariance function is identically zero.

\subsection{A geometrical approach}

In this section, the marked point process $\Phi$ is assumed to be stationary and simple, and the marks are assumed to be non-negative.

In the random field context, the value of a second-order characteristic at $r=0$ is obtained as a one-point quantity. For example, the value at the origin of the covariance function is the variance of the random field at a fixed single point. Following Capobianco and Renshaw (1998), a two-point characteristic should also be replaced by a one-point characteristic for $r=0$ in the case of a marked point process. Therefore, the reduced second moment measure is replaced by the non-reduced one in equation (2.1), i.e. $\sum^{\neq}$is substituted by $\sum$. The fact that only the distance between two points $x_{1}$ and $x_{2}$ is of interest can be taken into account directly, exchanging the indicator function $\mathbf{1}_{B_{2}}\left(x_{2}\right)$ in formula (2.1) by $\mathbf{1}_{I}\left(\left\|x_{2}-x_{1}\right\|\right)$, where $I \subset[0, \infty)$. We combine both aspects and replace $\alpha_{f}^{(2)}$ in (2.1) by $\mu_{f}^{(2 *)}$, where

$$
\mu_{f}^{(2 *)}\left(B_{1} \times I\right)=\mathrm{E} \sum_{\left[x_{1} ; m_{1}\right],\left[x_{2} ; m_{2}\right] \in \Phi} f\left(m_{1}, m_{2}\right) \mathbf{1}_{B_{1} \times I}\left(u\left(x_{1}, x_{2}\right)\right),
$$

$I \in \mathscr{B} \cap[0, \infty), B_{1} \in \mathscr{B}^{d}$ and $u\left(x_{1}, x_{2}\right)=\left(x_{1},\left\|x_{1}-x_{2}\right\|\right)$.

As the measure $\mu_{f}^{(2 *)}$ does not have a Lebesgue density, the function $\kappa_{f}^{*}$ is now necessarily defined as

$$
\kappa_{f}^{*}=\frac{\mathrm{d} \mu_{f}^{(2 *)}}{\mathrm{d} \mu^{(2 *)}} \quad \mu^{(2 *)} \text {-almost surely; }
$$

cf. (2.2) and (3.1). (Analogously to Section 2, the index $f$ is dropped if $f \equiv 1$.) As the marked point process is stationary, the function $\kappa_{f}^{*}$ depends on the second argument only.

Definition 3.1. The mark variogram, the mark covariance function, the $k_{m m}$-function, and the correlation function are defined for non-negative $r$ by equations (2.3), (2.4), (2.6), and (2.7), respectively, with $\kappa$ replaced by $\kappa^{*}$. The function $\kappa_{f}^{*}$ is given by equation (3.4) and is understood as a function depending on the second argument only.

The existing and new definitions coincide for positive arguments if the marked point process is isotropic and the second-order product density exists. This can be seen as follows. Let $r$ be a positive constant, $B_{1} \in \mathscr{B}^{d}$ and $B=\left\{\left(x_{1}, x_{2}\right): x_{1} \in B_{1}\right.$, $\left.0<\left\|x_{1}-x_{2}\right\|<r\right\}$. Then, by (3.3) and (2.1),

$$
\mu_{f}^{(2 *)}\left(B_{1} \times(0, r)\right)=\alpha_{f}^{(2)}(B) .
$$


Let $\mathrm{d} v^{(2)}(x, r)=r^{d-1} \rho^{(2)}(r) \mathrm{d} x \mathrm{~d} r$; then the equalities (3.5), (3.4), (2.2) and a coordinate transformation yield

$$
\begin{aligned}
\int_{B_{1} \times(0, r)} \kappa_{f}^{*} \mathrm{~d} \mu^{(2 *)} & =\int_{B} \kappa_{f}\left(\left\|x_{1}-x_{2}\right\|\right) \rho^{(2)}\left(\left\|x_{1}-x_{2}\right\|\right) \mathrm{d} x_{1} \mathrm{~d} x_{2} \\
& =\int_{B_{1} \times(0, r)} \kappa_{f}(r) \mathrm{d} v^{(2)}(x, r)
\end{aligned}
$$

for all Borel sets $B_{1} \in \mathscr{B}^{d}$ and all $r>0$. As both $\kappa_{f}$ and $\kappa_{f}^{*}$ are identically 1 for $f \equiv 1$ by definitions (2.2) and (3.4), the measures $\mu^{(2 *)}$ and $v^{(2)}$ are identical on $\mathscr{B}^{d} \otimes(\mathscr{B} \cap(0, \infty))$ by (3.6). It follows that $\kappa_{f}^{*}=\kappa_{f}\left(\mu^{(2 *)}\right.$-almost surely) for an arbitrary measurable function $f$ using (3.6) again.

\subsection{Estimators}

Estimators for the characteristics given in Definition 3.1 can easily be constructed. For positive arguments, known estimators for the characteristics given in Section 2.1 can be used, even in the case of an anisotropic marked point process. Baddeley (1999) gives a comprehensive overview of such estimators involving different edge corrections and kernel smoothers. However, for $r=0$, estimators based on one-point quantities are used. A simple example for an estimator is given by way of illustration.

Let $A$ be a sampling window in $\mathbb{R}^{2}$ and $|A|$ the area of $A$. Define $A+s=\{s+x: x \in A\}$ for $s \in \mathbb{R}^{2}$, and let $f: \mathbb{R}^{2} \rightarrow \mathbb{R}$ be a fixed function. The points of the point process within the window $A$ are denoted by $x_{i}$ and the corresponding marks are denoted by $m\left(x_{i}\right)$ for $i=1, \ldots, N$. Let $\delta$ be a suitably small positive number; then an estimator $\hat{\kappa}_{f}^{*}$ of $\kappa_{f}^{*}$ is given by

$$
\hat{\kappa}_{f}^{*}(h)= \begin{cases}\frac{1}{N} \sum_{i=1}^{N} f\left(m\left(x_{i}\right), m\left(x_{i}\right)\right), & h=0, \\ \sum_{\substack{i, j=1 \\ i \neq j}}^{N} \frac{f\left(m\left(x_{i}\right), m\left(x_{j}\right)\right) \mathbf{1}_{h-\delta<\left\|x_{i}-x_{j}\right\| \leqslant h+\delta}}{\left|\left(A+x_{i}\right) \cap\left(A+x_{j}\right)\right|}\left(\sum_{\substack{i, j=1 \\ i \neq j}}^{N} \frac{\mathbf{1}_{h-\delta<\left\|x_{i}-x_{j}\right\| \leqslant h+\delta}}{\left|\left(A+x_{i}\right) \cap\left(A+x_{j}\right)\right|}\right)^{-1}, \quad h>0 .\end{cases}
$$

The estimator for a specific second-order characteristic is now obtained by choosing suitable functions $f_{i}$ and composing the estimators $\hat{\kappa}_{f_{i}}^{*}$ according to the formulae given in Section 2. For positive arguments, the estimators obtained are identical to the estimators given in Cressie (1993, p. 716).

\subsection{A theoretical approach}

Here, the definitions are given in terms of a certain $\sigma$-algebra. They will allow for negative marks and extensions to more general Polish point spaces and real mark spaces. Yet they will 
agree with the definitions given in Section 3.2 if the marked point process is simple, stationary and if the marks are non-negative.

The new approach is motivated by the simple observation that the function $u$ in formula (3.3) defines a sub- $\sigma$-algebra of the Borel $\sigma$-algebra $\mathscr{B}^{2 d}$. The function $u$ itself will be ignored and only the sub- $\sigma$-algebra considered. For the sake of generality and for ease of notation, this sub- $\sigma$-algebra will be embedded in a larger $\sigma$-algebra, called $\mathscr{S}^{2}$.

Let $\Psi$ be an arbitrary marked (with mark space $\mathbb{M}$ and $\sigma$-algebra $\mathscr{C}$ ) or unmarked point process on $\mathbb{R}^{d}$. Let $\Psi^{n}$ be the point process whose realizations consist of $n$-vectors of identical components $(\psi, \ldots, \psi)$, where $\psi$ is a realization of $\Psi$. Then the nth moment measure $\mu^{(n)}$ of $\Psi$ is the intensity measure of $\Psi^{n}$, i.e.

$$
\mu^{(n)}(X)=\mathrm{E} \Psi^{n}(X)
$$

where $X \in\left(\mathscr{B}^{d} \otimes \mathscr{C O}\right)^{n}$ or $X \in \mathscr{B}^{d n}$.

In the following, $\Phi$ is an arbitrary, not necessarily stationary or simple, marked point process where the $n$th moment measure of the corresponding unmarked point process is $\sigma$ finite. For convenience' sake, $\Phi$ is supposed to be a marked point process on $\mathbb{R}^{d}$ and the marks are real-valued. Denote the product space of the point space $\mathbb{R}^{d}$ and the mark space $\mathbb{R}$ by $\mathbb{S}$, and let $\mathscr{S}$ be its product Borel- $\sigma$-algebra.

The subsequent function $h_{\mathscr{C}}$ constitutes the basis for the definition of an $n$ th-order characteristic. Let $\mathscr{C}$ be a sub- $\sigma$-algebra of the product $\sigma$-algebra $\mathscr{S}^{n}$, and let $\mu_{\mathscr{C}}^{(n)}$ be the restriction of $\mu^{(n)}$ to $\mathscr{C}$. Assume that $h$ is a $\mu^{(n)}$-integrable or positive and measurable function with domain $\mathbb{S}^{n}$. Then, the $\mathscr{C}$-measurable function $h_{\mathscr{C}}$ is defined by

$$
\mathrm{E} \int_{X} h(s) \mathrm{d} \Phi^{n}(s)=\int_{X} h_{\mathscr{C}}(s) \mathrm{d} \mu_{\mathscr{C}}^{(n)}(s) \quad \text { for all } X \in \mathscr{C} .
$$

(Equality (3.7) is justified by the Radon-Nikodym theorem, as $\mathrm{E} \int{ }_{(\cdot)} h(s) \mathrm{d} \Phi^{n}(s)$ is absolutely continuous with respect to $\mu^{(n)}$.)

Let $\Phi$ be a simple marked point process and $n=2$; then the left-hand side of equation (3.7) reduces to

$$
\mathrm{E} \sum_{\left[x_{1} ; m_{1}\right],\left[x_{2} ; m_{2}\right] \in \Phi} h\left(\left[x_{1} ; m_{1}\right],\left[x_{2} ; m_{2}\right]\right) \mathbf{1}_{X}\left(\left[x_{1} ; m_{1}\right],\left[x_{2} ; m_{2}\right]\right), \quad X \in \mathscr{C} .
$$

Compare (3.8) with the right-hand side of (3.3), where $f$ takes the role of $h$. A function $u$ is included in (3.3), whereas in (3.8) the set $X$ is required to be an element of $\mathscr{C}$.

For $B \in \mathscr{B}^{d}$ and positive $R$, let

$$
C_{B, R}=\left\{\left(\left[x_{1} ; m_{1}\right],\left[x_{2} ; m_{2}\right]\right): x_{1} \in B,\left\|x_{1}-x_{2}\right\| \leqslant R, m_{1} \in \mathbb{R}, m_{2} \in \mathbb{R}\right\}
$$

and let $\mathscr{C}$ be the $\sigma$-algebra generated by $\left\{C_{B, R}: B \in \mathscr{B}^{d}, R>0\right\}$. If $\Phi$ is stationary and the marks are non-negative, then the function $h_{\mathscr{C}}$ on the right-hand side of (3.7) coincides with $\kappa_{f}^{*}$ for $h\left(\left[x_{1} ; m_{1}\right],\left[x_{2} ; m_{2}\right]\right)=f\left(m_{1}, m_{2}\right)$. That is to say, (3.8) equals $\mu_{f}^{(2 *)}(B,[0, R])$ for $X=C_{B, R}$. Then, (3.4) and (3.7) yield 


$$
\int_{B \times[0, R]} \kappa_{f}^{*} \mathrm{~d} \mu^{(2 *)}=\int_{C_{B, R}} h_{\mathscr{C}} \mathrm{d} \mu_{\mathscr{C}}^{(2)}
$$

and, in the same way as for (3.6), it can be concluded that $\kappa_{f}^{*}$ equals $h_{\mathscr{C}}$ if both are understood as functions that depend on the distance only.

Definition 3.2. Let $\mathscr{Z}$ be an ensemble of bounded non-overlapping sets of $\mathbb{R}^{d}$ with positive Lebesgue measure that cover $\mathbb{R}^{d}$. Fix an arbitrary element $Z_{0}$ of $\mathscr{Z}$ and let

$$
\begin{aligned}
M\left(\left[x_{1} ; m_{1}\right]\right) & =m_{1}, \\
E\left(\left[x_{1} ; m_{1}\right],\left[x_{2} ; m_{2}\right]\right) & =m_{1}, \\
C\left(\left[x_{1} ; m_{1}\right],\left[x_{2} ; m_{2}\right]\right) & =m_{1} m_{2}, \\
V\left(\left[x_{1} ; m_{1}\right],\left[x_{2} ; m_{2}\right]\right) & =m_{1}^{2}, \\
\mathscr{D} & =\sigma(\mathscr{D} \times\{\varnothing, \mathbb{R}\})
\end{aligned}
$$

and

$\mathscr{C}=\sigma\left(\left\{\left\{\left(\left[x_{1} ; m_{1}\right],\left[x_{2} ; m_{2}\right]\right): x_{1} \in Z,\left\|x_{1}-x_{2}\right\| \leqslant R, m_{1} \in \mathbb{R}, m_{2} \in \mathbb{R}\right\}: Z \in \mathscr{Z}, R>0\right\}\right)$.

For $x_{1} \in Z_{0}, x_{2} \in \mathbb{R}^{d}$, and $s=\left(\left[x_{1} ; \cdot\right],\left[x_{2} ; \cdot\right]\right)$, let

$$
\begin{array}{rlrl}
E^{*}\left(\left\|x_{1}-x_{2}\right\|\right) & =E_{\mathscr{C}}(s) & & \left(\mu_{\mathscr{C}}^{(2)} \text { almost surely }\right), \\
C^{*}\left(\left\|x_{1}-x_{2}\right\|\right) & =C_{\mathscr{C}}(s) & & \left(\mu_{\mathscr{C}}^{(2)} \text { almost surely }\right), \\
V^{*}\left(\left\|x_{1}-x_{2}\right\|\right) & =V_{\mathscr{C}}(s) & \left(\mu_{\mathscr{C}}^{(2)} \text { almost surely }\right), \\
\bar{m}^{*} & =M_{\mathscr{D}}\left(x_{1}\right) . &
\end{array}
$$

Then, the functions $\gamma^{*}, \operatorname{cov}^{*}, k_{m m}^{*}$, and $\operatorname{cor}^{*}$ are defined on $[0, \infty)$ as

$$
\begin{aligned}
\gamma^{*}(r) & =V^{*}(r)-C^{*}(r), \\
\operatorname{cov}^{*}(r) & =C^{*}(r)-\left(E^{*}(r)\right)^{2}, \\
k_{m m}^{*}(r) & =\left(\bar{m}^{*}\right)^{-2} C^{*}(r), \\
\operatorname{cor}^{*}(r) & =\frac{C^{*}(r)-\left(E^{*}(r)\right)^{2}}{V^{*}(r)-\left(E^{*}(r)\right)^{2}} .
\end{aligned}
$$

Note that the functions on the left-hand side of (3.10)-(3.12) are well defined as the functions on the right-hand side are constant for $\left\|x_{1}-x_{2}\right\|=$ const. and $x_{1} \in Z_{0}$. The function $M_{\mathscr{D}}$ is constant on $Z_{0}$. 
The ensemble $\mathscr{E}$ can be interpreted as a set of sampling windows that cover $\mathbb{R}^{d}$, and $Z_{0}$ is the sampling window one is actually interested in. In the case of a stationary point process, the particular choice of the sampling window does not matter, since two functions of a characteristic that are defined on different windows $Z_{0}$ and $Z_{1}$ are identical (almost everywhere). Equation (3.7) and the subsequent comments show that the above definitions coincide with those given in Section 3.2 if the marked point process is stationary and simple and if the marks are non-negative. Definition 2 is also sound for non-stationary marked point processes as averaging a function over a bounded area remains feasible.

The new definitions do not lead to characteristics that are able to deal with directional features of anisotropic point processes. However, by using a different $\sigma$-algebra in (3.9), any directional property can be taken into account. For example, the $\sigma$-algebra $\mathscr{C}$,

$$
\begin{aligned}
\mathscr{C}= & \sigma\left(\left\{\left\{\left(\left[x_{1} ; m_{1}\right],\left[x_{2} ; m_{2}\right]\right): x_{1} \in Z, x_{1}-x_{2}=r(\cos \alpha, \sin \alpha), r \leqslant R,\right.\right.\right. \\
& \left.\left.\left.\alpha \in(-\pi / 4, \pi / 4] \cup(3 \pi / 4,5 \pi / 4], m_{1} \in \mathbb{R}, m_{2} \in \mathbb{R}\right\}: Z \in \mathscr{Z}, R>0\right\}\right),
\end{aligned}
$$

and the corresponding characteristics enable the handling of two main directions of anisotropy.

\subsection{Properties}

The following relations hold if $\Phi$ is simple:

$$
\gamma^{*}(0)=0, \quad \operatorname{cor}^{*}(0)=1, \quad V^{*}(0)=C^{*}(0) .
$$

If $\Phi$ is stationary, then

$$
E^{*}(0)=\bar{m} \equiv M_{\mathscr{D}} .
$$

If additionally, suitable mixing conditions are satisfied then

$$
E^{*}(0)=E^{*}(\infty), \quad C^{*}(\infty)=\bar{m}^{2}, \quad V^{*}(0)=V^{*}(\infty), \quad k_{m m}^{*}(\infty)=1 .
$$

\section{Examples}

In order to illustrate the characteristics defined in Section 3, two examples from Isham (1985) and Wälder and Stoyan $(1996 ; 1997)$ are reconsidered. In the following, $U\left(r, R_{1}, R_{2}\right)$ is the volume of the union of two spheres with radii $R_{1}$ and $R_{2}$ whose centres are a distance $r$ apart; the volume of the $d$-dimensional unit sphere is denoted by $b_{d}$.

Example 4.1. The mark variogram $\gamma^{*}$ of the following marked point process is a conditionally negative definite function, and both the mark covariance function $\operatorname{cov}^{*}$ and the mark correlation function cor* are positive definite. However the properties of the function $k_{m m}^{*}$ imply that the marked point process is not a random field model (3.2). 
Let $\Pi$ be a stationary Poisson process on $\mathbb{R}^{d}$ with intensity $\lambda$ and, for a fixed radius $R$, let the mark of a point $x \in \Pi$ equal the number of points in the sphere $b(x, R)$ excluding $x$ itself. If $a(r, R)=2 b_{d} R^{d}-U(r, R, R)$, then

$$
\begin{aligned}
\gamma^{*}(r) & =\lambda b_{d} R^{d}-\lambda a(r, R), \quad r \geqslant 0, \\
\operatorname{cov}^{*}(r) & =\lambda a(r, R), \quad r \geqslant 0, \\
k_{m m}^{*}(r) & =1+\frac{\lambda a(r, R)+\left(2 \lambda b_{d} R^{d}+1\right) \mathbf{1}_{0<r \leqslant R}}{\left(\lambda b_{d} R^{d}\right)^{2}}, \quad r \geqslant 0, \\
\operatorname{cor}^{*}(r) & =b_{d}^{-1} a(r / R, 1), \quad r \geqslant 0 .
\end{aligned}
$$

The function $k_{m m}^{*}$ has jump discontinuities both at the origin and at $r=R$ which can be explained by the jump discontinuities of the function $E^{*}(r)=\lambda b_{d} R^{d}+\mathbf{1}_{0<r \leqslant R}$. As $k_{m m}^{*}(0+)>k_{m m}^{*}(0)$, the function $k_{m m}^{*}$ cannot be positive definite; see Sasvári (1994, Theorem 1.4.1).

The graphs of $k_{m m}^{*}$ and cov $^{*}$ are shown in Figure 1 for $d=2, \lambda=1$ and $R=1$.

Example 4.2. Let $\Pi$ be a stationary Poisson process on the plane and let the mark $m(x)$ of a point $x \in \Pi$ be the distance between $x$ and its nearest neighbour in $\Pi$. If $\Phi$ is the error function, i.e. $\Phi(x)=2 \pi^{-1 / 2} \int_{0}^{x} \exp \left(-t^{2}\right) \mathrm{d} t$, then

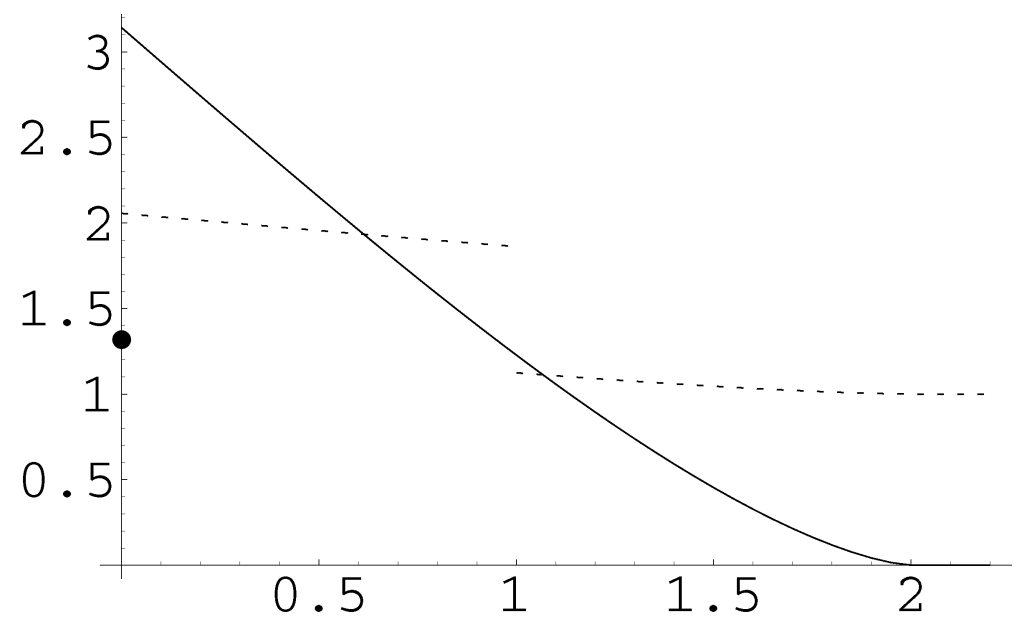

Figure 1. The functions $k_{m m}^{*}$ (dashed curve and single point) and $\operatorname{cov}^{*}$ (solid curve) of Example 4.1 with $\lambda=1, R=1$ and $d=2$. 


$$
\begin{aligned}
\gamma^{*}(r) & =\frac{1-\mathrm{e}^{-\lambda \pi r^{2}}}{\lambda \pi}-\int_{0}^{r} \int_{0}^{r} \mathrm{e}^{-\lambda U(r, s, t)} \mathrm{d} s \mathrm{~d} t, \\
\operatorname{cov}^{*}(r) & =\int_{0}^{r} \int_{0}^{r} \mathrm{e}^{-\lambda U(r, s, t)} \mathrm{d} s \mathrm{~d} t-\frac{\left\{\Phi\left((\lambda \pi)^{1 / 2} r\right)\right\}^{2}}{4 \lambda}+\frac{4-\pi}{4 \lambda \pi} \mathbf{1}_{r=0}, \\
k_{m m}^{*}(r) & =4 \lambda \int_{0}^{r} \int_{0}^{r} \mathrm{e}^{-\lambda U(r, s, t)} \mathrm{d} s \mathrm{~d} t+\frac{4}{\pi} \mathbf{1}_{r=0}, \\
\operatorname{cor}^{*}(r) & =\frac{\lambda \int_{0}^{r} \int_{0}^{r} \mathrm{e}^{-\lambda U(r, s, t)} \mathrm{d} s \mathrm{~d} t-\left\{\Phi\left((\lambda \pi)^{1 / 2} r\right)\right\}^{2} / 4}{\left(1-\mathrm{e}^{-\lambda \pi r^{2}}\right) / \pi-\left\{\Phi\left((\lambda \pi)^{1 / 2} r\right)\right\}^{2} / 4} \mathbf{1}_{r>0}+\mathbf{1}_{r=0} .
\end{aligned}
$$

Figure 2 shows the graphs of these functions for $\lambda=1$. Notice that the values of the functions $\gamma^{*}$ and $\operatorname{cov}^{*}$ have been multiplied by 20 .

Some properties of the functions are worth mentioning. Clearly, cov* is not a positive definite function, although cor* possibly is and $\gamma^{*}$ might be conditionally negative definite. Furthermore, there is a remarkable difference between the value of $\operatorname{cov}^{*}$ at the origin and its local maximum around 0.8 . The functions $\gamma^{*}(r), k_{m m}^{*}(r)$ and $\operatorname{cov}^{*}(r)$ tend to zero as $r \rightarrow 0$ since the marks of two points a distance $r$ apart have values between 0 and $r$. The fact that $\operatorname{cor}^{*}(r)$ tends to a positive value as $r \rightarrow 0$ is somewhat surprising.

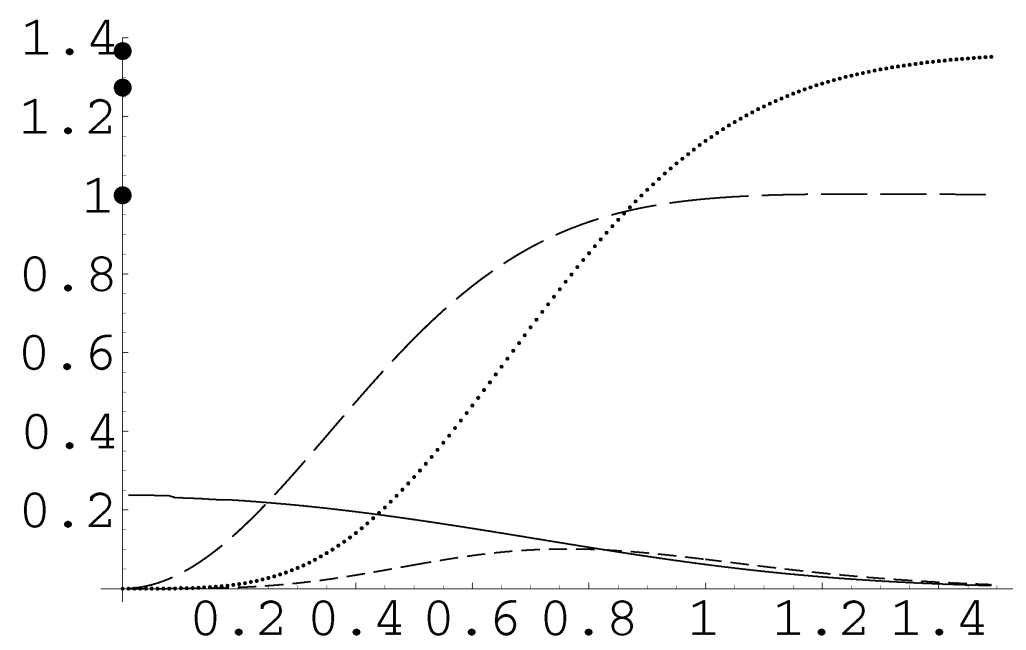

Figure 2. The functions $k_{m m}^{*}$ (long dashed curve and point $(0,1.273)$ ), cor ${ }^{*}$ (solid curve and point $(0,1)), 20 \gamma^{*}$ (dotted curve), and 20cov* (short dashed curve and point $(0,1.366)$ ) of Example 4.2 with $\lambda=1$. 


\section{Proof and extension of Theorems 2.1 and 2.2}

The following Theorem 5.1 shows that there is a marked point process whose characteristics $E^{*}, C^{*}$ and $V^{*}$ (see Definition 3.2) equal the functions $E, C$ and $V$, respectively, on a finite interval $(0, R]$ if the functions $E, C$ and $V$ are measurable and satisfy the inequalities

$$
0 \leqslant V-E^{2} \text { and }\left|C-E^{2}\right| \leqslant V-E^{2} .
$$

These inequalities guarantee that, for each $r \in(0, R]$, the terms $V(r)-E(r)^{2}$ and $C(r)-E(r)^{2}$ define a proper variance and covariance, respectively. Thus the above conditions for $E, C$ and $V$ cannot be weakened.

Theorems 2.1 and 2.2 are consequences of Theorem 5.1. As the assertion of Theorem 5.1 is fairly general, Theorems 2.1 and 2.2 can be seen purely as special cases of the former, so that they are corollaries rather than assertions in their own right. Further assertions like those given in Theorem 2.2, where the $k_{m m}$-function or the mark correlation function is replaced by another second-order characteristic, can also be obtained by Theorem 5.1.

Theorem 5.1. Let $R$ be a positive finite number, and let $C, E$ and $V$ be measurable real functions on $(0, \infty)$ such that

$$
E^{2} \leqslant V, \quad C \leqslant V, \quad 2 E^{2}-C \leqslant V .
$$

Assume that $m \equiv E$, if $E$ is constant, and

$$
m \in\left(\inf _{0<r \leqslant R}(E(r)), \sup _{0<r \leqslant R}(E(r))\right),
$$

otherwise. Then, for any dimension $d \geqslant 1$, we have the following:

(a) There exists a stationary and isotropic marked point process $\Phi$ on $\mathbb{R}^{d}$ such that, for almost all $r \in(0, R]$,

$$
E^{*}(r)=E(r), \quad C^{*}(r)=C(r) \quad V^{*}(r)=V(r) ;
$$

and for almost all $r>R$,

$$
E^{*}(r)=\bar{m}=m \quad \text { and } \quad C^{*}(r)=\bar{m}^{2} .
$$

(b) If, additionally, $E$ and $C$ are non-negative functions and if, for any positive $r$, the equality $E(r)=0$ implies that both $C(r)$ and $V(r)$ are 0 , then $\Phi$ can be chosen such that the marks are non-negative and the conditions in part (a) are satisfied.

The idea of the proof is to define a point process where each realization consists of two points only. For two given points a distance $r$ apart, random marks are easily constructed such that the respective second-order characteristics have the desired values. That is to say, the three inequalities in Theorem 5.1 guarantee that $\sigma^{2}=V(r)-E(r)^{2} \geqslant 0$ and that $|\tau| \leqslant \sigma^{2}$ with $\tau=C(r)-E(r)^{2}$. Then at least a bivariate normal distribution with expectation $E(r)$, variance $\sigma^{2}$, and correlation $\tau / \sigma^{2}$ exists.

An analogous stationary point process is obtained by a modified Matérn hard-core 
process consisting of suitably chosen pairs of points. For the Matern hard-core processes, see Stoyan et al. (1995) and the references therein.

Proof of Theorem 5.1. (a) The idea is to define a modified Matérn hard-core process of type I, called $\Phi$, by means of a certain marked Poisson process $\Pi$. Based on $\Phi$, a marked point process $\Psi$ consisting of pairs of points will be defined that satisfies the conditions in Theorem 5.1.

The assumption on $m$ implies that there exists a probability measure $P$ with $\operatorname{supp}(P)=(0, R]$ and $P(\{0\})=0$, such that

$$
\int_{(0, R]} E(r) \mathrm{d} P(r)=\bar{m}=m .
$$

Denote the uniform distribution on the sphere $S_{d-1}$ by $U\left(S_{d-1}\right)$. Let $\Pi$ be a stationary marked Poisson point process with independent and identically distributed marks. A mark $m$ consists of the tuple $\left(\alpha, \xi, \zeta_{1}, \zeta_{2}\right)$, where $\alpha, \xi, \zeta_{1}$ and $\zeta_{2}$ are independent random variables and

$$
\alpha \sim U\left(S_{d-1}\right), \quad \xi \sim P, \quad \zeta_{1} \sim N(0,1), \quad \xi_{2} \sim N(0,1) .
$$

The marked point process $\Phi$ consists of all the points $\left[x ;\left(\alpha, \xi, \zeta_{1}, \zeta_{2}\right)\right] \in \Pi$ for which the union of the spheres $b(x, 2 R) \cup b(x+R \alpha, 2 R)$ contains no further (unmarked) point of $\Pi$ except $x$ itself. The marks of $\Pi$ are carried over. The marked point process $\Psi$ is defined in the following way. Each point $\left[x_{1} ;\left(\alpha, \xi, \zeta_{1}, \zeta_{2}\right)\right] \in \Phi$ gets a neighbour $x_{2}$ whose position depends on the variables $\alpha$ and $\xi: x_{2}=x_{1}+\xi \alpha$. The marks of $x_{1}$ and $x_{2}$ are real numbers and are denoted by $u_{1}$ and $u_{2}$, respectively. They depend on $\xi, \xi_{1}$, and $\xi_{2}$ : let $\sigma^{2}=$ $V(\xi)-(E(\xi))^{2}$ and $\tau=C(\xi)-(E(\xi))^{2}$. Let $A(s, t)$ be a symmetric $2 \times 2$ matrix depending measurably on $s$ and $t$ such that $A^{2}(s, t)=\left(\begin{array}{c}s t \\ t s\end{array}\right)$. Then, the marks $u_{1}$ and $u_{2}$ are defined as

$$
\left(\begin{array}{l}
u_{1} \\
u_{2}
\end{array}\right)=A\left(\sigma^{2}, \tau\right)\left(\begin{array}{l}
\zeta_{1} \\
\zeta_{2}
\end{array}\right)+\left(\begin{array}{l}
E(\xi) \\
E(\xi)
\end{array}\right) .
$$

Hence, the second-order characteristics of $\Psi$ are defined for almost all $r>0$. They satisfy the conditions in Theorem 5.1 for values $r \leqslant R$ by construction of the pairs of marked points. For $r>R$, the marks are independent; thus, $C^{*}(r)=E^{*}(r)^{2}$ and $E^{*}(r)=\bar{m}$ by (5.1).

(b) If both $E$ and $C$ are non-negative and if the equality $E(r)=0$ implies $C(r)=V(r)=0$, then the above construction of the locations is kept. However, a mark of $\Pi$ consists now of the tuple $\left(\alpha, \xi, \zeta_{1}, \zeta_{2}, \zeta_{3}\right)$ where $\alpha, \xi, \zeta_{1}, \zeta_{2}$ and $\zeta_{3}$ are independent and

$$
\alpha \sim U\left(S_{d-1}\right), \quad \xi \sim P, \quad \xi_{1} \sim U(0,1), \quad \xi_{2} \sim U(0,1), \quad \xi_{3} \sim U(0,1) .
$$

Here $U(0,1)$ is the uniform distribution on the interval $(0,1)$. The marks of $\Psi$ will be such that, for a fixed pair of points at distance $\xi \leqslant R$, the marks $u_{1}$ and $u_{2}$ take only two different, non-negative, values, namely $a=a(\xi)$ and $b=b(\xi)$.

Let $e=E(\xi), c=C(\xi)$ and $v=V(\xi)$ and define 


$$
\begin{aligned}
& p= \begin{cases}1 / 2, & \text { if } v=e^{2}, \\
\left(\min \left\{\frac{v}{v-e^{2}}, 2\right\}\right)^{-1}, & \text { otherwise }\end{cases} \\
& a=e-\left(v-e^{2}\right)^{1 / 2}(1-p)^{1 / 2} p^{-1 / 2}, \\
& b=e+\left(v-e^{2}\right)^{1 / 2} p^{1 / 2}(1-p)^{-1 / 2},
\end{aligned}
$$

and

$$
u_{1}=a \mathbf{1}_{\zeta_{1} \leqslant p}+b \mathbf{1}_{\zeta_{1}>p} .
$$

It follows from the definition of $p$ that $1 / 2 \leqslant p<1$, i.e. $p \geqslant 1-p>0$. The value of $b$ is clearly non-negative and the value of $a$ satisfies

$$
a=e-\left(v-e^{2}\right)^{1 / 2}(1-p)^{1 / 2} p^{-1 / 2}=\max \left\{0, e-\left(v-e^{2}\right)^{1 / 2}\right\} \geqslant 0 .
$$

If $c \geqslant e^{2}$, define

$$
r= \begin{cases}1, & \text { if } v=e^{2} \\ \frac{c-e^{2}}{v-e^{2}}, & \text { otherwise }\end{cases}
$$

and

$$
u_{2}=u_{1} \mathbf{1}_{\zeta_{2} \leqslant r}+\left(a \mathbf{1}_{\zeta_{3} \leqslant p}+b \mathbf{1}_{\zeta_{3}>p}\right) \mathbf{1}_{\zeta_{2}>r}
$$

If $c<e^{2}$, define

$$
r=\frac{\left(e^{2}-c\right) p}{\left(v-e^{2}\right)(1-p)}
$$

and

$$
u_{2}=\left(b \mathbf{1}_{\zeta_{1} \leqslant 1-p}+a \mathbf{1}_{\zeta_{1}>1-p}\right) \mathbf{1}_{\zeta_{2} \leqslant r}+\left(a \mathbf{1}_{\zeta_{3} \leqslant p}+b \mathbf{1}_{\zeta_{3}>p}\right) \mathbf{1}_{\zeta_{2}>r} .
$$

If $v=e^{2}$ then the third inequality of Theorem 5.1 yields $c \geqslant e^{2}$. Thus $v \neq e^{2}$ in (5.3), and $r$ is well defined. The value of $r$ is given by (5.2) or (5.3) lies in the interval $[0,1]$. This is clear for $c \geqslant e^{2}$; for $c<e^{2}$, the definition of $p$ yields

$$
r=\frac{e^{2}-c}{\min \left\{e^{2}, v-e^{2}\right\}},
$$

which is also in $[0,1]$.

The definitions of $u_{1}$ and $u_{2}$ yield $\mathrm{E} u_{1}=\mathrm{E} u_{2}=e$ and $\mathrm{E} u_{1}^{2}=\mathrm{E} u_{2}^{2}=v$. If $c \geqslant e^{2}$ then

$$
\mathrm{E} u_{1} u_{2}=r \mathrm{E} u_{1}^{2}+(1-r)\left(\mathrm{E} u_{1}\right)^{2}=r v+(1-r) e^{2}=c,
$$

using the definition (5.2) of $r$. If $c<e^{2}$ then

$$
\mathrm{E} u_{1} u_{2}=r\left((2 p-1) a^{2}+2(1-p) a b\right)+(1-r) e^{2}
$$


as $p \geqslant 1-p$. The definitions of $a, b$, and $r$ yield

$$
\mathrm{E} u_{1} u_{2}=e^{2}-p^{-1}(1-p)\left(v-e^{2}\right) r=c .
$$

Hence the pairwise marks $u_{1}$ and $u_{2}$ are non-negative and have the required characteristics $E(\xi), V(\xi)$ and $C(\xi)$. The construction of the locations leads, as in the case of non-positive marks, to a marked point process with the stated properties.

Remark. Theorem 5.1 does not state that, for any given unmarked stationary point process, there exist stationary marks such that the equalities $E^{*}=E, V^{*}=V$ and $C^{*}=C$ hold on $(0, R]$, if the functions $E, V$ and $C$ obey the inequalities in Theorem 5.1.

A counterexample in $\mathbb{R}$ is the stationary lattice process with lag 1 . This point process can be reinterpreted as a stationary process on $\mathbb{Z}$. Thus the possible second-order characteristics for the marks are essentially only the positive definite functions on $\mathbb{Z}$. See Sasvári (1994, Sections 1.10 and 3.1) for more details.

Proof of Theorem 2.1. Let $f_{+}$and $f_{-}$be the positive and the negative part of $f$, respectively. In order to prove assertions (a), (b) and (c), Theorem 5.1 is applied with

(a) $E=\left(1+f_{-}\right)^{1 / 2}, C=1+f_{+}, V=1+2|f|$;

(b) $E \equiv m=1, C=1+f, V=1+|f|$; and

(c) $E \equiv m=1, C=1+f, V \equiv 2$.

The assertion for $\gamma$ is proved by choosing $C \equiv E \equiv m=1$ and $V=1+f$ in Theorem 5.1.

The proof of Theorem 2.1(b) shows also that there are stationary and isotropic marked point processes (with real-valued marks) such that $k_{m m}=f+1$ for positive arguments if $f$ is any fixed real-valued measurable function on $(0, \infty)$ with compact support.

Proof of Theorem 2.2. Define

$$
E_{1}^{*}(r)= \begin{cases}(f(r) / 2)^{1 / 2}, & \text { if } 0<r \leqslant R \\ \sqrt{2}, & \text { if } R<r \leqslant R+1, \\ 1 / \sqrt{2}, & \text { if } R+1<r \leqslant R+2 \\ 1, & \text { if } R+2<r\end{cases}
$$

and

$$
E_{2}^{*}(r)= \begin{cases}(3 f(r) / 2)^{1 / 2}, & \text { if } 0<r \leqslant R, \\ 1 / \sqrt{2}, & \text { if } R<r \leqslant R+1, \\ \sqrt{2}, & \text { if } R+1<r \leqslant R+2, \\ 1, & \text { if } R+2<r .\end{cases}
$$

Let

$$
V_{1}^{*}(r)= \begin{cases}2 E_{1}^{*}(r)^{2}+E_{2}^{*}(r)^{2}+f(r)+1, & \text { if } 0<r \leqslant R, \\ 22, & \text { if } R<r \leqslant R+1, \\ 10.5, & \text { if } R+1<r \leqslant R+2\end{cases}
$$


and

$$
V_{2}^{*}(r)= \begin{cases}E_{1}^{*}(r)^{2}+2 E_{2}^{*}(r)^{2}+f(r)+1, & \text { if } 0<r \leqslant R, \\ 10.5, & \text { if } R<r \leqslant R+1, \\ 22, & \text { if } R+1<r \leqslant R+2 .\end{cases}
$$

Finally, define $C_{1}^{*}=C_{2}^{*}=f$ and $\bar{m}=1$. By Theorem 5.1 (replacing $R$ by $R+2$ ), there exist two marked point processes $\Phi_{j}, j=1,2$, with characteristics $E_{j}^{*}, V_{j}^{*}, C_{j}^{*}$ and $\bar{m}$. Furthermore, $\operatorname{cor}^{(1)}=-\operatorname{cor}^{(2)}$, and $\operatorname{cor}^{(1)}(r) \neq 0$ for $r \in(0, R+2]$. The definitions of $E_{i}^{*}, C_{i}^{*}$ and $V_{i}^{*}$ are such that the marks of $\Phi_{1}$ and $\Phi_{2}$ can be chosen non-negative.

Assume now that $k_{m m}(r) \leqslant 0$ for a certain $r \in(0, R]$. If $k_{m m}(r)<0$ then the mark correlation function cor is necessarily negative at $r$ by (2.6) and (2.7), or (3.13) and (3.14). For any two non-negative random variables $X$ and $Y$ with $\mathrm{E} X Y=0$ it follows that the correlation $\operatorname{cov}(X, Y)(\operatorname{var} X \operatorname{var} Y)^{-1 / 2}$ is negative or that $X$ or $Y$ equals zero almost surely. In the latter case, the correlation is not defined. Thus, $\operatorname{cor}(r)$ can never be non-negative, if $k_{m m}(r)=0$ and if the marks of the point process are non-negative.

The proof of Theorem 2.2 shows that the following corollary also holds.

Corollary 5.3. Let $R$ be a finite positive number and let $f(r)$ be a measurable function on $(0, \infty)$ that is identically 1 for $r>R$. Then, the function $f$ is non-negative if and only if there exist two stationary and isotropic marked point processes $\Phi_{1}$ and $\Phi_{2}$ with real-valued marks such that (2.8) and (2.9) hold and that $\operatorname{cor}^{(1)}(r) \neq 0$ if $f(r) \neq 0$ and $0<r \leqslant R$.

\section{Acknowledgement}

The author is grateful to Dietrich Stoyan for drawing the author's attention to the work of Isham (1985) and for many comments that improved an earlier version of the paper. Peter Diggle and Paulo Ribeiro contributed many valuable hints and stimulating discussions. An anonymous referee inspired the investigation of marked point processes with non-negative marks, which led to the sharpening of several assertions. This work has been supported by the EU Training and Mobility of Researchers network on 'Statistical and computational methods for the analysis of spatial data' (ERB-FMRX-CT96-0095).

\section{References}

Baddeley, A.J. (1999) Spatial sampling and censoring. In O.E. Barndorff-Nielsen, W.S. Kendall and M.N.M. van Lieshout (eds), Stochastic Geometry, Likelihood and Computation, pp. 37-78. Boca Raton, FL: Chapman \& Hall/CRC.

Baddeley, A.J., Møller, J.M. and Waagepetersen, R. (1998) Non- and semi-parametric estimation of interaction in inhomogeneous point patterns. Technical Report 12, Biometry Research Unit, Danish Institute of Agricultural Sciences, Tjele. 
Capobianco, R. and Renshaw, E. (1998) The autocovariance function for marked point processes: a comparison between two different approaches. Biometrical J., 40, 431-446.

Cressie, N.A.C. (1993) Statistics for Spatial Data. New York: Wiley.

Diggle, P.J. (1983) Statistical Analysis of Point Processes. London: Chapman \& Hall.

Diggle, P.J., Lange, N. and Beneš, F. (1991) Analysis of variance for replicated spatial point patterns in clinical neuroanatomy. J. Amer. Statist. Assoc., 86, 618-625.

Gavrikov, V.L. and Stoyan, D. (1995) The use of marked point processes in ecological and environmental forest studies. Environ. Ecological Statist., 2, 331-344.

Goulard, M., Pages, L. and Cananettes, A. (1995) Marked point processes - using correlation functions to explore a spatial data set. Biometrical J., 37, 837-853.

Isham, V. (1985) Marked point processes and their correlations. In F. Droesbeke (ed.), Spatial Processes and Spatial Time Series Analysis, pp. 63-75. Brussels: Publications des Facultés Universitaires Saint-Louis.

Mase, S. (1996) The threshold method for estimating total rainfall. Ann. Inst. Statist. Math., 48, 201213.

Penttinen, A.K. and Stoyan, D. (1989) Statistical analysis for a class of line segment processes. Scand. J. Statist., 16, 153-168.

Penttinen, A.K., Stoyan, D. and Henttonen, H.M. (1992) Marked point processes in forest statistics. Forest Sci., 38, 806-824.

Ripley, B.D. (1977) Modelling spatial patterns. J. Roy. Stat. Soc. Ser. B, 39, 172-192.

Ripley, B.D. (1981) Spatial Statistics. New York: Wiley.

Sasvári, Z. (1994) Positive Definite and Definitizable Functions. Berlin: Akademie Verlag.

Stoyan, D. (1984a) Correlations of the marks of marked point processes - statistical inference and simple models. Elektron. Informationsverarbeitung Kybernetik, 20, 285-294.

Stoyan, D. (1984b) On correlations of marked point processes. Math. Nachr., 116, 197-207.

Stoyan, D. (1990) Stereological formulae for a random system of nonintersecting spheres. Statistics, 21, $131-236$.

Stoyan, D. and Stoyan, H. (1994) Fractals, Random Shapes and Point Fields. Chichester: Wiley.

Stoyan, D., Kendall, W.S. and Mecke, J. (1995) Stochastic Geometry and its Applications, 2nd edition. Chichester: Wiley.

Wackernagel, H. (1995) Multivariate Geostatistics. Berlin: Springer-Verlag.

Wälder, O. and Stoyan, D. (1996) On variograms in point process statistics. Biometrical J., 38, 895905.

Wälder, O. and Stoyan, D. (1997) Models of marking and thinning of Poisson processes. Statistics, 29, $179-202$.

Wälder, O. and Stoyan, D. (1998) On variograms in point process statistics: Erratum. Biometrical J., 40, 109.

Wen, R. and Sinding-Larsen, R. (1997) Stochastic modelling and simulation of small faults by marked point processes and kriging. In E.Y. Baafi and N.A. Schofield (eds), Geostatistics Wollongong '96, Vol. 1, pp. 398-414. Dordrecht: Kluwer.

Received October 1998 and revised May 2000 